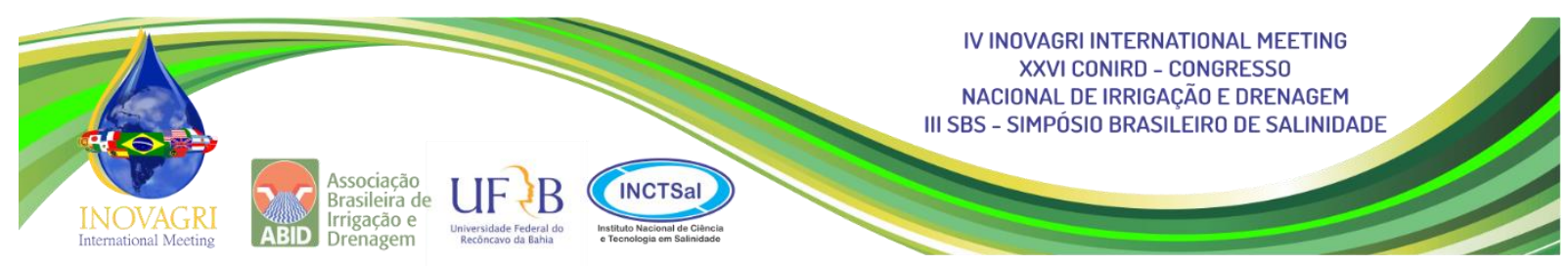

\title{
AVALIAÇÃO DA UNIFORMIDADE DE UM SISTEMA DE IRRIGAÇÃO POR GOTEJAMENTO.
}

\author{
M. H. C. Souza ${ }^{1}$; R. D. S. Santos ${ }^{1}$; L. H. Bassoi ${ }^{2}$.
}

RESUMO: Um sistema de irrigação eficiente, aliado ao manejo adequado, pode garantir o suprimento apropriado de água a cultura. Dessa forma a avaliação dos sistemas de irrigação se torna um procedimento indispensável para garantir um bom desempenho da cultura no campo. A baixa eficiência do sistema pode afetar diretamente a produtividade, pois a baixa uniformidade de distribuição pode favorecer o desenvolvimento espacialmente diferenciado da cultura. Desta forma o objetivo do trabalho foi avaliar a uniformidade de distribuição de água em sistemas de irrigação por gotejamento no cultivo de videira de vinho. O teste foi realizado no Campo Experimental de Bebedouro, pertencente à Embrapa Semiárido, na cidade de Petrolina-PE (latitude S 09 08'08,09', longitude O 40 18'33,6' e altitude 373m). Foram avaliadas as uniformidades de distribuição de água no sistema de irrigação por gotejamento, utilizando 96 gotejadores, sendo feitas avaliações em todas as linhas laterais, observando o primeiro emissor, o localizado a 1/3 e o localizado a 2/3 do início da linha, e o último emissor da linha. Após coleta dos dados, foram calculadas as respectivas vazões e posteriormente determinado o coeficiente de uniformidade de distribuição (CUD), o coeficiente de uniformidade de Christiansen (CUC), o coeficiente de uniformidade estatístico (CUE), o coeficiente de uniformidade de Hart (CUH), o coeficiente de variação (CV) e eficiência de aplicação (EA). O CUD obteve uma classificação como "razoável”, já os demais coeficientes CUC (94,2\%), CUH (92,5\%) e CUE (90,6\%) foram classificados como "excelente". O CV $(9,38 \%)$ foi classificado como "razoável" e a EA obteve-se valor de 82,0\% classificada como "aceitável". Observou-se que este sistema apresenta ótima uniformidade, apesar da variabilidade nas vazões.

PALAVRAS-CHAVE: coeficiente de uniformidade, coeficiente de variação, eficiência de aplicação.

\section{EVALUATION OF THE UNIFORMITY OF A DRIP IRRIGATION SYSTEM.}

\footnotetext{
${ }^{1}$ Doutorando (a) em Irrigação e Drenagem, UNESP/FCA, CEP: 18.610-307, campus Botucatu, SP. Fone: (14) 3880-7100. Email: marcello_henry@hotmail.com

${ }^{2}$ Pesquisador EMBRAPA Instrumentação São Carlos.
} 
SUMMARY: An efficient irrigation system, together with proper water management, can ensure the proper supply of water to the crop. In this way the evaluation of the irrigation systems becomes an indispensable procedure to guarantee a good crop development in the field. The low efficiency of the system can directly affect yield, since the low uniformity of water distribution can favor the spatially differentiated development of the crop. In this way the objective of the work was to evaluate the uniformity of water distribution in drip irrigation system in a wine grape orchard. The test was carried out at the Experimental Field of Bebedouro, belonging to Embrapa Semi-arid, Petrolina, state of Pernambuco, Brazi (latitude S $09^{\circ} 08$ '08,09' ', longitude $\mathrm{O} 40^{\circ} 18$ ' 33,6 " and altitude $373 \mathrm{~m}$ ). The water distribution uniformity was evaluated in the drip irrigation system, using 96 drippers, and evaluations were made on all the lateral lines, observing the first emitter, one located at $1 / 3$ and other located at $2 / 3$ of the line beginning, and the last emitter of the line. After data collection, the respective flow rates were calculated and the distribution uniformity coefficient (CUD), Christiansen uniformity coefficient (CUC), coefficient of statistical uniformity (CUE), Hart uniformity coefficient $(\mathrm{CUH})$, coefficient of variation (CV) and application efficiency (EA) were determined. The CUD obtained a classification as "reasonable", while the other coefficients CUC (94.2\%), CUH (92.5\%) and CUE (90.6\%) were classified as "excellent". The CV (9.4\%) was classified as "reasonable" and the EA (82.0\%) was classified as "acceptable". It was observed that this system presents an excellent uniformity, despite the variability in the flow rates.

KEYWORDS: coefficient of uniformity, coefficient of variation, application efficiency.

\section{INTRODUÇÃO}

Um sistema de irrigação eficiente, aliado ao manejo adequado, pode garantir o suprimento apropriado de água a cultura. Segundo Santos et al., (2015) a irrigação por gotejamento é o sistema mais apropriado a economia de água e energia, possibilidade de automação e fertirrigação e que está em notável expansão. Entretanto tal sistema é considerado susceptível ao entupimento dos emissores, caso não haja uma avaliação e/ou manutenção periódica adequada.

Uma informação importante que deve se ter conhecimento na avaliação de sistemas de irrigação localizada, tanto na fase de projeto como no acompanhamento do desempenho após a implantação, é a uniformidade de distribuição (FAVETTA \& BOTREL, 2001). Dessa forma a 
avaliação dos sistemas de irrigação se torna um procedimento indispensável para garantir uma redução nos gastos da irrigação, assim como o bom desempenho da cultura no campo.

A baixa eficiência do sistema pode afetar diretamente a produtividade agrícola, pois a baixa uniformidade de distribuição pode favorecer um desenvolvimento espacialmente diferenciado da cultura. Esse fato ocorre, principalmente, devido à localização da água na zona radicular das culturas, em pequenas intensidades, porém com alta frequência.

De forma a evitar o baixo rendimento das culturas, devido aos problemas na uniformidade de aplicação da água, é necessário verificar periodicamente o sistema de irrigação, buscando manter as condições recomendadas no projeto. Isso só é possível a partir da associação das medidas de uniformidade e conceitos de eficiência (VALNIR JUNIOR et al., 2013).

Souza et al. (2005) afirmam que o decréscimo na uniformidade de aplicação é causado geralmente por uma manutenção inapropriada do sistema de irrigação, gerando uma perda de aproximadamente $60 \%$ da uniformidade. Outros fatores que afetam a uniformidade é a variação na fabricação (nas características do gotejador) e o seu tempo de uso.

Para a determinação do coeficiente de uniformidade de distribuição de água em um sistema de irrigação por gotejamento, é necessário o conhecimento da vazão dos gotejadores, para emprego dessa informação em métodos que fornecem critérios de avaliação, como o coeficiente de uniformidade de distribuição (CUD) e coeficiente de uniformidade de Christiansen (CUC), o coeficiente de uniformidade estatístico (CUE), o coeficiente de uniformidade de Hart (CUH), o coeficiente de variação (CV) e eficiência de aplicação (EA).

Estas equações fornecem valores confiáveis, no entanto em grandes áreas são necessários muito tempo e mão-de-obra, devido a quantidade de emissores do sistema de gotejamento. No entanto, algumas metodologias verificam que 16 ou até 24 emissores é uma quantidade considerada como suficiente na determinação da uniformidade do sistema.

Com isso, objetivo do trabalho foi avaliar a uniformidade de distribuição da água em sistema de irrigação localizada por gotejamento em pomar de videira por meio da determinação de diferentes coeficientes de uniformidade de distribuição da água.

\section{MATERIAL E MÉTODOS}

O trabalho foi realizado no Campo Experimental de Bebedouro, pertencente à Embrapa Semiárido, na cidade de Petrolina-PE (latitude S 090 08' 08,09', longitude O $40^{\circ} 18^{\prime}$ '33,6” e altitude $373 \mathrm{~m}$ ). Segundo a classificação de Koppen, o clima da região apresenta-se como tropical semiárido, tipo BSwh', caracterizado pela escassez e irregularidade nas precipitações, 
com chuvas no verão e elevada evaporação, exigindo assim de aplicações complementares de água as culturas implantadas na região pela irrigação.

$\mathrm{Na}$ área encontra-se implantado um pomar de uvas viníferas cultivar Syrah sobre portaenxerto Paulsen 1103, estabelecido no ano de 2009, com as plantas sendo conduzidas no sistema de espaldeira, com três fios de arame no espaçamento de $1 \mathrm{~m}$ entre plantas e de $3 \mathrm{~m}$ entre fileiras,e constituindo um total de 30 fileiras e 24 plantas por fileiras ( 720 plantas no total). $\mathrm{O}$ sistema de irrigação implantado e avaliado foi o gotejamento, com um emissor a cada $0,5 \mathrm{~m}$ na fileira de plantas, totalizando 2 emissores por planta, com pressão de serviço de $100 \mathrm{KPa}$ e vazão média aferida de $2,5 \mathrm{~L} \mathrm{~h}^{-1}$.

A determinação da uniformidade de distribuição de água do sistema de gotejamento teve como parâmetro a metodologia de Keller \& Karmeli (1974). Assim, foi avaliado a uniformidade de distribuição de água no sistema de irrigação por gotejamento, utilizando 96 gotejadores, sendo feitas avaliações em todas as linhas laterais, observando o primeiro emissor, o segundo localizado a 1/3, o terceiro a 2/3 do início e o último emissor da linha.

A determinação da vazão foi realizada por meio da coleta com recepientes do volume de água aplicado pelo emissor durante o período de dois minutos, e medição do volume por meio de uma proveta graduada. A verificação da pressão de serviço ocorreu por meio do uso de um manômetro instalado na tubulação de saída de água do sistema de bombeamento, que encontrase a $20 \mathrm{~m}$ a área em avaliação.

De posse dos dados coletados, foram calculadas as respectivas vazões e posteriormente determinado o coeficiente de uniformidade de distribuição - CUD (Equação 1); o coeficiente de uniformidade de Christiansen - CUC (Equação 2); o coeficiente de uniformidade estatístico - CUE (Equação 3); coeficiente de uniformidade de Hart - CUH (Equação 4), coeficiente de variação - CV (Equação 5) e eficiência de aplicação - EA (Equação 6) conforme as equações abaixo.

$$
\mathrm{CUD}=100 \cdot \frac{\overline{\mathrm{X}}_{25}}{\overline{\mathrm{X}}}
$$

Em que:

CUD: Coeficiente de Uniformidade de Distribuição;

$\bar{X}_{25}$ : Média das $25 \%$ menores descargas dos emissores, em $\mathrm{L} \mathrm{h}^{-1}$;

$\bar{X}$ : Média das descargas de todos os emissores, em $\mathrm{L} \mathrm{h}^{-1}$.

$$
\mathrm{CUC}=100 \cdot\left[1-\frac{\sum_{\mathrm{i}=1}^{\mathrm{n}}|\mathrm{Xi}-\overline{\mathrm{X}}|}{\mathrm{n} \cdot \overline{\mathrm{X}}}\right]
$$

Em que:

CUC: Coeficiente de Uniformidade de Christiansen; 
n: Número de observações;

$\mathrm{X}_{\mathrm{i}}$ : Lâmina de água coletada no i-ésimo ponto sobre a superfície do solo;

$\bar{X}$ : Lâmina média aplicada.

$$
\mathrm{CUE}=100 *\left(1-\frac{\mathrm{S}_{\mathrm{d}}}{\mathrm{Q}_{\text {méd }}}\right)
$$

Em que:

CUE: Coeficiente de Uniformidade Estatístico;

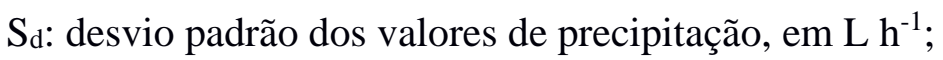

qméd: Média das vazões coletadas nos gotejadores na subárea, em $\mathrm{L} \mathrm{h}^{-1}$.

$$
\mathrm{CUH}=\left[1-\sqrt{\frac{2}{\pi}} * \frac{\mathrm{S}_{\mathrm{d}}}{\mathrm{q}_{\mathrm{méd}}}\right] * 100
$$

Em que:

CUH: Coeficiente de Uniformidade de Hart, em \%;

$\mathrm{S}_{\mathrm{d}}$ d desvio padrão dos valores de precipitação, $\mathrm{em} \mathrm{L} \mathrm{h}^{-1}$;

qméd: Média das vazões coletadas nos gotejadores na subárea, em $\mathrm{L} \mathrm{h}^{-1}$.

$$
\mathrm{CV}=\frac{\mathrm{S}_{\mathrm{d}}}{\mathrm{q}_{\mathrm{méd}}} * 100
$$

Em que:

CV: Coeficiente de Variação, em \%;

$\mathrm{S}_{\mathrm{d}}$ : desvio padrão dos valores de precipitação, $\mathrm{em} \mathrm{L} \mathrm{h}^{-1}$;

qméd: Média das vazões coletadas nos gotejadores na subárea, em $\mathrm{L} \mathrm{h}^{-1}$.

$$
\mathrm{E}_{\mathrm{a}}=0,9 * \mathrm{CUD}
$$

Em que:

Ea: Eficiência de aplicação;

CUD: Coeficiente de Uniformidade de Distribuição.

A metodologia para avaliação da uniformidade de irrigação no sistema de gotejamento foi fundamentada nos índices CUC (CHRISTIANSEN, 1942), CUD (CRIDDLE et al., 1956), CUE, CUH, CV e EA. A interpretação dos valores de CUC, CUD, CUE e CV baseou-se na proposição de Mantovani (2001), Frizzone et al. (2012) e os padrões da ASAE EP 405.1 (2003), citado por Borssoi et al. (2012), e apresentadas nas Tabela 1.

A eficiência de aplicação de água foi classificada conforme metodologia de Bralts (1986) citado por Bernardo et al. (2006), na qual os valores (\%) de EA $\geq 95$ são considerados como ideal; $80 \leq$ EA < 95 são considerados como aceitável, EA $<80$ são considerados como inaceitável. 


\section{RESULTADO E DISCUSSÃO}

Para o sistema de irrigação por gotejamento em avaliação, os valores e classificação do CUD, CUC, CUE e CUH, obtidos foram, respectivamente, de 73,8 \% (razoável), 94,2 \%, 90,6 $\%$ (excelente) e $92,5 \%$ (excelente), enquanto o CV foi de 9,4\% (razoável) e a EA foi de 82,0 \% (aceitável), de acordo com Mantovani (2001) e Frizzone et al. (2012) e 9,38\% de acordo com os padrões da ASAE EP 405.1 (2003), citado por Borssoi et al. (2012).

Segundo Keller \& Bliesner (1990) citado por Santos et al. (2015), o CUD é o coeficiente mais utilizado em avaliação de sistemas, devido a sensibilidade para perceber variações na distribuição de água de um sistema de irrigação, pois considera a razão entre a média do menor quartil e a lâmina média coletada, o que favorece a possibilidade de maior atenção as plantas que recebem uma menor lâmina de irrigação.

Baixos valores do CUD podem representar perda de água por percolação profunda quando a lâmina mínima aplicada corresponde à lâmina necessária ou ainda desuniformidade dos emissores, e se a cultura apresentar potencial econômico e sistema radicular raso, esse valor deve ser superior a 80\% (PAULINO et al., 2009; SANTOS et al., 2012; SANTOS et al., 2015).

De acordo com Borssoi et al. (2012), quanto menor o CV, significa dizer que houve uma menor dispersão quando comparado a média e consequentemente melhor será a distribuição uniforme de água, resultando numa menor variabilidade espacial entre as lâminas.

Já os coeficientes CUC, CUE e CUH foram classificados como excelente de acordo com Mantovani (2001) e Frizzone et al. (2012) (94,16; 90,62 e 92,52\%, respectivamente). Em sistemas de irrigação por gotejamento deve-se considerar um limite mínimo aceitável de $80 \%$ no valor do CUC. Portanto, esta área está relacionada ao conceito de área adequadamente irrigada (CUNHA et al, 2009).

Conforme Valnir Junior et al. (2013) o valor mínimo para CUC é de 90\%, o mesmo cita que Mantovani et al. (2009), consideram que valores acima de $84 \%$ já podem ser considerados adequados para sistemas de irrigação. Corroborando com a classificação relatada nesse estudo.

Vale ressaltar que o CUC é um índice baseado no desvio médio absoluto, para expressar a dispersão das lâminas e o CUH e CUE são baseados no desvio-padrão como medida de dispersão, dessa forma valores semelhantes são esperados e assim demonstram que há distribuição normal referente as lâminas aplicadas pelos emissores (SANTOS et al., 2015).

A EA calculada foi de $82,03 \%$, sendo classificada como aceitável, porém segundo Mantovani et al. (2009) e corroborado por Santos et al. (2015), a EA deve estar entre 90 a 95\% para se caracterizar como valores recomendados para um sistema de irrigação. 


\section{CONCLUSÕES}

O sistema de irrigação por gotejamento avaliado apresenta bons índices de uniformidade e eficiência, entretanto ainda são necessários ajustes no sistema, tendo em vista que o CUD e a EA foram abaixo da classificação ideal (excelente).

\section{REFERÊNCIAS BIBLIOGRÁFICAS}

ASAE EP 405.1 (2003). In: BORSSOI, A. L., VILAS BOAS, M. A., REISDÖRFER, M., HERNÁNDEZ, R. H., FOLLADOR, F. A. Water application uniformity and fertigation in a dripping irrigation set. Engenharia Agrícola, v. 32, n. 4, p. 718-726, 2012.

BERNARDO, S.; SOARES, A.A.; MANTOVANI, E.C. Manual de irrigação. 8 ed. Viçosa: UFV, Imprensa Universitária, 2006. 625p.

BORSSOI, A. L., VILAS BOAS, M. A., REISDÖRFER, M., HERNÁNDEZ, R. H., FOLLADOR, F. A. Water application uniformity and fertigation in a dripping irrigation set. Engenharia Agrícola, v. 32, n. 4, p. 718-726, 2012.

BRALTS, V. F. Field performance and evaluation. In: NAKAYAMA, Francis S.; BUCKS, D. A. (Ed.). Trickle irrigation for crop production: design, operation and management. Elsevier, 2012.

CHRISTIANSEN, J.E. Irrigation by sprinkler. Berkeley: California Agricultural Station, 1942. $212 p$.

CRIDDLE, W.D.; DAVIS, S.; PAIR, C.H.; SHOCKELEY, D.G. Methods for evaluating irrigation systems. Washington: SCS-USDA, 1956. 24p.

CUNHA, F. F., ALENCAR, C. A. B., VICENTE, M. R., BATISTA, R. O., SOUZA, J. A. R. Comparação de equações para cálculo da uniformidade de aplicação de água para diferentes sistemas de irrigação. Engenharia na agricultura, v. 17, n. 5, 2009.

FAVETTA, G. M. \& BOTREL, T. A. Uniformidade de sistemas de irrigação localizada: validação de equações. Sci. Agric.. vol.58 no.2 Piracicaba Apr/June 2001.

FRIZZONE, J.A.; FREITAS, P.D.; REZENDE, R.; FARIA, M.D. Microirrigação: gotejamento e microaspersão. Maringá: Eduem, 2012. 
KELLER \& BLIESNER (1990). In: SANTOS, M. A. L., SANTOS, D. P., SILVA, D. S., SANTOS SILVA, M., CAVALCANTE, P. H. S. Avaliação da uniformidade de distribuição de um sistema de irrigação por gotejamento em inhame (Dioscoreacayennensis L.). Revista Ciência Agrícola, Ciência Agrícola, Rio Largo, v. 13, n. 1, p. 7-13, 2015.

KELLER, J. \& KARMELI, D. Trickle irrigation design parameters. Transactions of the ASAE, v. 17 , n. 4, p. $678-0684,1974$.

MANTOVANI, E. C. AVALIA: Programa de Avaliação da Irrigação por Aspersão e Localizada. Viçosa, MG: UFV, 2001.

MANTOVANI, E. C., BERNARDO, S., PALARETTI, L. F. Irrigação - Princípios e Métodos. Editora UFV. Pág. 355, 3ª ed. Viçosa - MG, 2009.

PAUlinO, M. A. O., DE FIGUEIREDO, F. P., FERNANDES, R. C., MAIA, J. T. L. S., DE OLIVEIRA GUILHERME, D., \& BARBOSA, F. S. (2013). Avaliação da uniformidade e eficiência de aplicação de água em sistemas de irrigação por aspersão convencional- Revista Brasileira de Agricultura Irrigada v.3, n.2, p.48-54, 2009

SANTOS, D. D. O., FREIRE, F. G. C., SANTOS, F. S. S., SANTOS, M. M. S., DE S LIMA, R. M., SANTOS, W. O. Avaliação da uniformidade de aplicação de água na irrigação por microaspersão na cultura da banana em São João do Jaguaribe-CE. In: I Inovagri International Meeting \& IV Winotec. Fortaleza - CE. 2012. Disponível em: http://www.inovagri.org.br/meeting2012/wp-content/uploads/2012/06/Protocolo061.pdf Acesso em: mar. 2017.

SANTOS, M. A. L., SANTOS, D. P., SILVA, D. S., SANTOS SILVA, M., CAVALCANTE, P. H. S. Avaliação da uniformidade de distribuição de um sistema de irrigação por gotejamento em inhame (Dioscoreacayennensis L.). Revista Ciência Agrícola, Ciência Agrícola, Rio Largo, v. 13, n. 1, p. 7-13, 2015.

SOUZA, L. O. C.; MANTOVANI, E. C.; SOARES, A. A.; RAMOS, M. M.; FREITAS, P. S. L. Avaliação de Sistemas de Irrigação por Gotejamento, Utilizados na Cafeicultura. Revista Brasileira de Engenharia Agrícola e Ambiental. v.10, n.3, p.541-548. Campina Grande, PB, DEAg/UFCG. 2005.

VALNIR JUNIOR, M. CARVALHO, C. M., SANTOS NETO, A. M., SOARES, J. I., LIMA, S. C. R. V., CARVALHO, M. A. R. Análise de desempenho em laboratório de linha gotejadora 
antes e após sua utilização em campo. DOI: 10.7127/rbai. v5n400068. REVISTA BRASILEIRA DE AGRICULTURA IRRIGADA-RBAI, v. 5, n. 4, 2013.

Tabela 1. Classificação do CUD, CUC, CUE e CV para sistemas de irrigação por gotejamento.

\begin{tabular}{ccccc}
\hline Classificação & CUD (\%) & CUC (\%) & CUE (\%) & CV (\%) \\
\hline Excelente & $>90$ & $90-100$ & $90-100$ & $<5$ \\
Bom & $80-90$ & $80-90$ & $80-90$ & $5-7$ \\
Razoável & $70-80$ & $70-80$ & $70-80$ & $7-11$ \\
Ruim & $<70$ & $60-70$ & $60-70$ & $11-15$ \\
Inaceitável & - & - & $<60$ & $>15$ \\
\hline
\end{tabular}

Fonte: Adaptado de Mantovani (2001), Frizzone et al. (2012) e Borssoi et al. (2012). 